\title{
BOUNDARY BEHAVIOR OF UNIVALENT FUNCTIONS SATISFYING A HÖLDER CONDITION
}

\author{
MATTS ESSÉN
}

\begin{abstract}
Let $f$ be univalent in the unit disk $U$ and continuous in $U \cup T$, where $T=\partial U$. We prove that if $f$ satisfies a Hölder condition, then each point in $f(T)$ is the image of at most finitely many points on $T$. The bound for the number of preimages depends in a sharp way on the Hölder exponent.
\end{abstract}

Let $U$ be the unit disk and $T=\partial U$ be the unit circle. G. Piranian has asked the following question: Assume that $f$ is analytic and univalent in $U$ and continuous in $U \cup T$. Furthermore, assume that for some positive $\varepsilon$ and some constant $C$, we have

$$
\left|f\left(z_{1}\right)-f\left(z_{2}\right)\right| \leqslant C\left|z_{1}-z_{2}\right|^{e+2 / 3}, \quad z_{1}, z_{2} \in U \cup T .
$$

Is it true that every point in $f(T)$ is the image of at most two points on $T$ ?

The answer to Piranian's question is in fact yes and is a consequence of the case $p=3$ in the following more general result.

THEOREM. Let $p \geqslant 3$ be an integer and suppose that $a$ is given and that $a>2 / p$. Let $f$ be as above except that (1) is replaced by

$$
\limsup _{r \rightarrow 1-}(1-r)^{-a}\left|f\left(e^{i \theta}\right)-f\left(r e^{i \theta}\right)\right|<\infty, \quad|\theta|<\pi .
$$

Then every point in $f(T)$ is the image of at most $p-1$ points on $T$.

Proof. Let us assume that $0 \in f(T)$ is the image of $p$ different points $\left\{z_{k}\right\}_{1}^{p}$ on $T$. Then there exist $p$ disjoint regions $\left\{\Omega_{k}\right\}_{1}^{p}$, all with 0 as a boundary point, such that $f^{-1}\left(\Omega_{k}\right)$ is a neighborhood in $U$ of $z_{k}, k=1,2, \ldots, p$. We can also assume that the boundary of $\Omega_{k}$ is contained in $f(T) \cup\{w:|w|=R\}, k=1,2, \ldots, p$, where $R$ is a small positive number.

Let $u$ be the harmonic measure of the circle $\{w:|w|=R\}$ with respect to the set $f(U) \cap\{w:|w|<R\}$, i.e., the function which is harmonic in the set, 1 on $f(U) \cap\{w:|w|=R\}$ and 0 on $f(T) \cap\{w:|w|<R\}$ except possibly on a set of capacity zero. For $0<r<R$, we define $\sigma_{k}(r)=\sup u\left(r e^{i \theta}\right), r e^{i \theta} \in \Omega_{k}$. We now use a deep result of $M$. Heins (cf. (4.1) in [1, p. 111]) which implies that there exists an absolute constant $C$ such that

$$
\prod_{1}^{p} \sigma_{k}(r) \leqslant C^{p}(r / R)^{p^{2} / 2}, \quad 0<r<R .
$$

Received by the editors October 27, 1980.

1980 Mathematics Subject Classification. Primary 30C55.

(C) 1981 American Mathematical Society 0002-9939/81/0000-0419/\$01.50 
It follows that $\min _{k} \sigma_{k}(r) \leqslant C(r / R)^{p / 2}, 0<r<R$. We conclude that in at least one of the regions $\left\{\Omega_{k}\right\}_{1}^{p}$, say $\Omega$, there exists a sequence $\left\{r_{n}\right\}_{1}^{\infty}$ decreasing to zero such that

$$
\max _{r e^{i \theta} \in \Omega} u\left(r e^{i \theta}\right) \leqslant C(r / R)^{p / 2}, \quad r \in\left\{r_{n}\right\}_{1}^{\infty} .
$$

Let $z_{0} \in T$ be such that $f\left(z_{0}\right)=0$ and $f^{-1}(\Omega)$ is a neighborhood in $U$ of $z_{0}$. We shall study the harmonic function $v(z)=u(f(z))$ near $z_{0}$. It is harmonic and positive in $f^{-1}(\Omega)$ and vanishes on $T$ near $z_{0}$. It is well known that there exists a positive constant $c$ such that

$$
v(z) \geqslant c(1-|z|), \quad z \text { near } z_{0}
$$

(cf. Heins [2, (9.3)] or Protter and Weinberger [3, Theorem 7, p. 65]).

We define $\gamma_{n}=\left\{w:|w|=r_{n}\right\} \cap \Omega$ and $\Gamma_{n}=f^{-1}\left(\gamma_{n}\right) . \Gamma_{n}$ and parts of $T$ near $z_{0}$ form the boundary of a region $D_{n}:\left\{D_{n}\right\}_{1}^{\infty}$ is a decreasing sequence of sets. For each $n$, we choose $t_{n}<1$ such that $t_{n} z_{0} \in \Gamma_{n}$. For $z \in\left\{t_{n} z_{0}\right\}$ and for large indices $n$, we have

$$
\begin{aligned}
c(1-|z|) & \leqslant v(z)=u(f(z))<C R^{-p / 2}\left|f(z)-f\left(z_{0}\right)\right|^{p / 2} \\
& \leqslant C\left(R, z_{0}\right)(1-|z|)^{a p / 2} .
\end{aligned}
$$

In this chain of inequalities, we first used (4), then (3), and finally (2).

Thus $\left(1-t_{n}\right)^{1-a p / 2}$ is bounded from above as $n \rightarrow \infty$. Since $t_{n} \rightarrow 1$ as $n \rightarrow \infty$, this is possible only if $a p<2$. Our assumption that ap $>2$ now gives us a contradiction which proves the theorem.

EXAMPLe. Let $p \geqslant 3$ be given and consider the function $f(z)=$ $z^{-1}\left(\left(1-z^{p}\right)^{2} / 2\right)^{1 / p}$ which defines a univalent mapping of $U$ onto the complement in $\underline{C} \cup\{\infty\}$ of the set $\{w \in \underline{C}: 0 \leqslant|w|<1, \arg w=(1+2 k) \pi / p, k=$ $0,1, \ldots, p-1\}$ (the $p$ th root is defined as real when $z \in U$ is real). For this function, the origin in the $w$-plane is the image of $p$ points on $T$. If $z_{0} \in T$ is such that $z_{0}^{p}=1$, we have

$$
\left|f\left(r z_{0}\right)-f\left(z_{0}\right)\right|=r^{-1}\left(\left(1-r^{p}\right)^{2} / 2\right)^{1 / p} \approx C_{p}(1-r)^{2 / p}, \quad r \rightarrow 1-.
$$

For other points on $T$, there are better estimates. Thus an estimate of type (2) holds for $f$ with $a=2 / p$.

This function $f$ has a pole at the origin and is not analytic in $U$. However, it is now easy to construct a function analytic in $U$ which satisfies the estimates above. We omit the details.

I am grateful to H. S. Shapiro for telling me about the question of G. Piranian.

\section{REFERENCES}

1. M. Heins, On the Denjoy-Carleman-Ahlfors Theorem, Ann. of Math. 49 (1948), 533-537.

2. Selected topics in the classical theory of functions of a complex variable, Holt, Rinehart and Winston, New York, 1962.

3. M. H. Protter and H. F. Weinberger, Maximum principles in differential equations, Prentice-Hall, Englewood Cliffs, N. J., 1967.

Department of Mathematics, Royal Institute of Technology, S-10044 Stockholm, Sweden

Current address: Department of Mathematics, University of Uppsala, S-75238 Uppsala, Sweden 